\title{
Generalised block bootstrap and its use in meteorology
}

\author{
László Varga and András Zempléni \\ Department of Probability Theory and Statistics, Eötvös Loránd University, Budapest, Hungary \\ Correspondence to: László Varga (vargal4@ chello.hu)
}

Received: 6 June 2016 - Revised: 12 April 2017 - Accepted: 9 May 2017 - Published: 14 June 2017

\begin{abstract}
In an earlier paper, Rakonczai et al. (2014) emphasised the importance of investigating the effective sample size in case of autocorrelated data. The simulations were based on the block bootstrap methodology. However, the discreteness of the usual block size did not allow for exact calculations. In this paper we propose a new generalisation of the block bootstrap methodology, which allows for any positive real number as expected block size. We relate it to the existing optimisation procedures and apply it to a temperature data set. Our other focus is on statistical tests, where quite often the actual sample size plays an important role, even in the case of relatively large samples. This is especially the case for copulas. These are used for investigating the dependencies among data sets. As in quite a few real applications the time dependence cannot be neglected, we investigated the effect of this phenomenon on the used test statistic. The critical value can be computed by the proposed new block bootstrap simulation, where the block size is determined by fitting a VAR model to the observations. The results are illustrated for models of the used temperature data.
\end{abstract}

\section{Introduction}

In the last decades the bootstrap methodology has become more and more widespread in different areas of statistical applications. See, e.g., Chernick (2011) for a review of possible areas from spatial models to financial data and data mining, where bootstrap may be used. In this paper we focus on the effect of the serial dependence, naturally arising in many time series data. The bootstrap samples must match the dependence within the data, so the block bootstrap is the suggested method for bootstrapping time series. Hall et al. (1995) investigated this approach in some detail, including suggestions for selecting the optimal block size. In an earlier paper, Rakonczai et al. (2014) investigated the possibilities for using the block bootstrap methods for checking the validity of the copula models. In this paper we present an improvement to the classical block bootstrap methodology, which is especially relevant in our applications.

In Sect. 2, we first briefly review the importance of stationarity of time series. In the bivariate case, the vector autoregression (VAR) process is one of the most important models, becoming popular first in the area of econometrics (Sims, 1980). For recent applications of VAR models in meteorology, see for example Hill et al. (2014), Norrulashikin et al. (2015) or Farook and Kannan (2016). Norrulashikin et al. (2015) investigated the applicability of VAR models to monthly temperature, precipitation and wind speed data, and they found that in quite a few cases VAR(1) was among the best models. We briefly present the main properties of VAR models, which are used in the sequel and present the notations.

In Sect. 3, we introduce the concept of copulas, the most convenient objects for analysing the dependence structures among variables. Their history goes back as far as Hoeffding (1940), but their applications are much more recent. However, they have spread very quickly to the most important areas - for a recent analysis in meteorology, see Cong and Brady (2012). Schölzel and Friederichs (2008) provided an overview of the possible applications of copula models in meteorology, more specifically in joint analysis of temperature and precipitation data. Most of these works use different parametric copula models, but we are more interested in testing for possible changes in the dependency structure, so we also introduce the most recent approaches in testing homogeneity of such models, which are based on the empirical copula process.

Section 4 is devoted to the bootstrap resampling method, including the block bootstrap approach, which is suitable for 
the case of serially dependent observations. Here we introduce a generalisation, which helps overcoming the problem that originally the block size was supposed to be a natural number. In our approach the block size is a random variable with arbitrary positive real-valued expectation greater than 1 , and it contains the original block bootstrap as a special case. Due to this small variance in the sample size, it overcomes the problem of extensive random error in the case of the stationary bootstrap (Politis and Romano, 1994).

Section 5 shows the results of our simulations regarding the properties of the proposed homogeneity test. It turned out that it is consistent and it has reasonable power for relatively small sample sizes. We have also investigated the effect of the block size for the properties of the test.

In Sect. 6 we apply our approach to the gridded temperature data base of E-OBS, which is a product of the EU-FP6 project ENSEMBLES (Haylock et al., 2008). Here we use the daily mean temperature data from the $0.5^{\circ}$ grid. Our focus is on checking for possible changes in the dependence pattern between the grid point close to Budapest and some other grid points within the Carpathian Basin. We show that in some cases there is a significant deviation from homogeneity of the first and second part of the data. The application of bootstrap methods in the context of statistical inference for copulas is a recent but quickly expanding area. Kojadinovic and Yan (2011b) proposed a quick method for bootstrapping the $p$ values in goodness-of-fit tests. For us the most relevant paper is Bücher and Volgushev (2013), where the consistency of the block bootstrap method for the empirical copula is proven under general conditions.

The conclusion summarises our findings and gives some interesting open questions.

\section{Vector autoregression (VAR) processes}

We call the $d$-dimensional random variable series $\left\{\boldsymbol{X}_{t}\right\}_{t \in \mathbb{Z}}=$ $\left(\boldsymbol{X}_{0}, \boldsymbol{X}_{ \pm 1}, \boldsymbol{X}_{ \pm 2}, \ldots\right)$ a time series if its elements are $d$ dimensional random vectors, which are usually not independent from each other. Here we consider $t$ as the time. Let us assume that the random variables have finite second moments. The time series $\left\{\boldsymbol{X}_{t}\right\}_{t \in \mathbb{Z}}$ is weakly stationary (hereafter just called stationary), if neither the mean function $E\left(\boldsymbol{X}_{t}\right)$ nor the covariance matrix $\operatorname{Cov}\left(\boldsymbol{X}_{t+s}, \boldsymbol{X}_{t}\right)$ depends on $t$ for all $s \in \mathbb{Z}$. Stationarity is an important property, it means that translation invariance holds for its statistical properties (mean, variance, autocovariance structure, etc., depending on the specific notion of stationarity considered).

One of the most frequently applied time series models are the autoregressive (AR) processes and their multidimensional counterparts, vector autoregressive (VAR) models. In the following, we define the $\operatorname{VAR}(p)$ process and give its main properties in two dimensions as this is necessary to our applications.
The time series $\left\{\boldsymbol{X}_{t}\right\}_{t \in \mathbb{Z}}=\left\{\left(X_{1, t}, X_{2, t}\right)^{T}\right\}_{t \in \mathbb{Z}}$ is called a zero-mean two-dimensional $\operatorname{VAR}(p)$ process if

$\boldsymbol{X}_{t}=\mathbf{A}_{1} \boldsymbol{X}_{t-1}+\mathbf{A}_{2} \boldsymbol{X}_{t-2}+\ldots+\mathbf{A}_{p} \boldsymbol{X}_{t-p}+\boldsymbol{\varepsilon}_{t}$,

where $\mathbf{A}_{1}, \ldots, \mathbf{A}_{p}$ are $2 \times 2$ parameter matrices, the independent innovation process $\left\{\boldsymbol{\varepsilon}_{t}\right\}_{t \in \mathbb{Z}}$ is a two-dimensional white noise with $E\left(\boldsymbol{\varepsilon}_{t}\right)=\mathbf{0}=(0,0)^{T}$ and $\operatorname{Cov}\left(\boldsymbol{\varepsilon}_{t}\right)=C$ a symmetric positive definite covariance matrix. The $\operatorname{VAR}(p)$ process is stationary if the roots of the characteristic polynomial $P(x)=\operatorname{det}\left(\mathbf{I}_{2}-\mathbf{A}_{1} x-\ldots-\mathbf{A}_{p} x^{p}\right)$ lie outside the unit circle.

Any $\operatorname{VAR}(p)$ process can be rewritten as a $\operatorname{VAR}(1)$ process in the following way: $\mathbf{Z}_{t}=A \mathbf{Z}_{t-1}+\boldsymbol{e}_{t}$, where

$$
\mathbf{Z}_{t}=\left(\begin{array}{c}
\boldsymbol{X}_{t} \\
\boldsymbol{X}_{t-1} \\
\vdots \\
\boldsymbol{X}_{t-p+1}
\end{array}\right), \boldsymbol{e}_{t}=\left(\begin{array}{c}
\boldsymbol{\varepsilon}_{t} \\
\mathbf{0} \\
\vdots \\
\mathbf{0}
\end{array}\right) \text { and }
$$

$\mathbf{A}=\left(\begin{array}{ccccc}\mathbf{A}_{1} & \mathbf{A}_{2} & \cdots & \mathbf{A}_{p-1} & \mathbf{A}_{p} \\ \mathbf{I}_{2} & \mathbf{0} & \cdots & \mathbf{0} & \mathbf{0} \\ \mathbf{0} & \mathbf{I}_{2} & \cdots & \mathbf{0} & \mathbf{0} \\ \vdots & \vdots & \ddots & \vdots & \vdots \\ \mathbf{0} & \mathbf{0} & \cdots & \mathbf{I}_{2} & \mathbf{0}\end{array}\right)$.

This representation is convenient in calculating the autocovariances. An equivalent condition for stationarity is that all the eigenvalues of the coefficient matrix $\mathbf{A}$ are smaller than 1 in modulus. In this case, the time series has a causal representation in terms of an infinite moving average form $\mathbf{Z}_{t}=\sum_{i=0}^{\infty} A^{i} \boldsymbol{e}_{t-i}$.

For the remainder of this section we assume that $\boldsymbol{X}_{t}$ is stationary. Let us denote with $\Gamma_{X}(h)=E\left(\boldsymbol{X}_{1+h} \boldsymbol{X}_{1}^{T}\right)$ the autocovariance function of the process $\boldsymbol{X}_{t} \cdot \boldsymbol{\Gamma}_{X}(h)$ is a $2 \times 2$ matrixvalued function, the symbols $\gamma_{i, j}(h)$ stand for its elements. We denote with $\boldsymbol{\Gamma}_{Z}(h)=E\left(\mathbf{Z}_{1+h} \mathbf{Z}_{1}^{T}\right)$ the $2 p \times 2 p$ matrixvalued autocovariance function of the process $\mathbf{Z}_{t}$. The covariance matrix of $\mathbf{Z}_{t}$ is $\boldsymbol{\Gamma}_{Z}(0)$, which can be determined by solving the matrix equation $\boldsymbol{\Gamma}_{Z}(0)-A \boldsymbol{\Gamma}_{Z}(0) A^{T}=\operatorname{Cov}\left(\boldsymbol{e}_{t}\right)$. It is easy to see that for $1 \leq h \in \mathbb{Z}$, the autocovariances can be calculated by $\boldsymbol{\Gamma}_{Z}(h)=A^{h} \boldsymbol{\Gamma}_{Z}(0)$. The powers of the matrix $A$ can easily be computed using the spectral decomposition. Lastly, we need the autocovariance matrix of the original process, and by construction, it is the upper left $2 \times 2$ submatrix of $\boldsymbol{\Gamma}_{Z}(h)$.

In the applications we will use the covariance matrix of the sample mean. The following asymptotic result will be crucial in our investigations: if $\sum_{h=-\infty}^{\infty}\left|\gamma_{i, i}(h)\right|<\infty$ for $i=1,2$, then $n \cdot \operatorname{tr}\left(\operatorname{Cov}\left(\overline{\boldsymbol{X}}_{n}\right)\right) \longrightarrow \sum_{i=1}^{2} \sum_{h=-\infty}^{\infty} \gamma_{i, i}(h) \quad$ as $n \rightarrow \infty$,

where $\operatorname{tr}(\cdot)$ denotes the trace of a matrix. 
It is important to check if the chosen time series model is adequate. If the model fits well, the fitted residuals should behave as a realisation of a white noise process. The hard part is to check whether the residuals are independent, thus there is no serial dependence among them. There are several methods for verifying this property, the most standard is the LjungBox test, which tests whether a specified group (usually the first 10-20 lags) of autocorrelations is different from zero. Another often applied serial correlation test is the BreuschGodfrey test. A more recent multidimensional approach was published in Kojadinovic and Yan (2011a), where the test was based on the empirical copula process. The main ideas and the concept of this test stem from Genest and Rémillard (2004).

For further details about time series analysis, see, for example, Brockwell and Davis (2013) or Shumway and Stoffer (2011).

\section{Copulas and their goodness-of-fit}

Let $\quad \boldsymbol{X}=\left(X_{1}, \ldots, X_{d}\right)^{T}$ be a random vector with joint distribution function $F_{X}(\mathbf{x})=$ $F_{X_{1}, \ldots, X_{d}}\left(x_{1}, \ldots, x_{d}\right)$ and marginal distribution functions $F_{1}\left(x_{1}\right)=F_{X_{1}}\left(x_{1}\right), \ldots, F_{d}\left(x_{d}\right)=F_{X_{d}}\left(x_{d}\right)$. Sklar's theorem claims that there exists a copula $C$, a distribution over the $d$-dimensional unit cube, with uniform margins, such that

$F_{X_{1}, \ldots, X_{d}}\left(x_{1}, \ldots, x_{d}\right)=C\left(F_{1}\left(x_{1}\right), \ldots, F_{d}\left(x_{d}\right)\right)$

Moreover, the copula $C$ is unique if the marginal distribution functions are continuous. This construction allows for the investigation of the dependence structure without specifying the marginal distributions. In the recent literature, various families of copulas have been introduced; for an overview and examples see, e.g., the introductory textbook of Nelsen (2007).

In this paper we focus on testing the homogeneity of copulas, motivated by the question of whether climate change also has an effect on the dependence between pairs of temperature observations. If this change is indeed observable, then it may have a substantial effect on the spatial structure of temperature anomalies, worth further meteorological investigations. So we do not have to go into the parametric inference, as we are just interested in the homogeneity analysis.

Let us suppose we have two independent samples of $\mathbb{R}^{d}$ valued random vectors. The first sample is $\boldsymbol{X}_{1}, \ldots, \boldsymbol{X}_{n}$ and the second one is $\boldsymbol{Y}_{1}, \ldots, \boldsymbol{Y}_{m}$. Formally we intend to test the hypothesis that the dependence structure of the two copulas has arisen from the same copula $C_{0}$. The most obvious way for testing the homogeneity of two copulas is to consider multidimensional $\chi^{2}$ approaches, but in this case we need to discretise the data, losing valuable information. In order to avoid its use, we can follow the approach of Rémillard and Scaillet (2009), who have developed a method for this problem. Their approach is based on the empirical copula, defined for the first sample as

$C_{n}(\boldsymbol{u})=\frac{1}{n} \sum_{i=1}^{n} I\left(\boldsymbol{U}_{i} \leq \boldsymbol{u}\right)$,

where $\boldsymbol{u} \in \mathbb{R}^{d}$ and $\boldsymbol{U}_{i}$ denotes the $d$-dimensional vector of the rank-based pseudo-observations: $\boldsymbol{U}_{i}=\boldsymbol{U}_{i, n}=$ $\left(U_{i 1, n}, \ldots, U_{i d, n}\right)$, where $n$ refers to the size of the sample and $U_{i j, n}=\frac{n}{n+1} F_{j}\left(X_{i j}\right)$. For illustrations see Figure 1, which depicts the original data points (standardised temperature data) and the $\boldsymbol{U}_{i}$ pseudo-observations for the grid point pairs close to Budapest and Sopron, respectively. Similarly, based on the pseudo-observations $\boldsymbol{U}_{i}$ and $\boldsymbol{V}_{i}$ of the first and the second samples, respectively, we can define the empirical copulas $C_{1, n}(\boldsymbol{u})$ and $C_{2, m}(\boldsymbol{u})$ (where $n$ and $m$ denote the sizes of the samples).

The proposed tests for checking the homogeneity of two samples are based on functionals of the empirical process:

$\kappa_{n, m}(\boldsymbol{u})=\frac{C_{1, n}(\boldsymbol{u})-C_{2, m}(\boldsymbol{u})}{\sqrt{\frac{1}{n}+\frac{1}{m}}}$,

where the asymptotic properties of the statistic can be based on the limit of the empirical copula processes. There are two different kinds of approaches investigated in Genest et al. (2006): the Cramér-von Mises type statistic $S_{n, m}=$ $\int\left(\kappa_{n, m}(\boldsymbol{u})\right)^{2} d \boldsymbol{u}$, and the Kolmogorov-Smirnov type $[0,1]^{d}$

statistic $T_{n, m}=\sup _{\boldsymbol{u} \in[0,1]^{d}}\left|\kappa_{n, m}(\boldsymbol{u})\right|$. As the second approach is considered to be generally less powerful, we based our inference on the statistic $K_{*}=\frac{1}{N^{d}} \sum_{i_{1}, \ldots, i_{d}}\left(\kappa_{n, m}\left(t_{i_{1}}, \ldots, t_{i_{d}}\right)\right)^{2}$, where $\left(t_{i_{j}}\right)_{i_{j}=1}^{N}, j=1, \ldots, d$ are appropriately fine divisions of the interval $(0,1)$. After some calculations, the Cramérvon Mises test statistic can be written in the following form (see Rémillard and Scaillet, 2009):

$$
\begin{aligned}
& S_{n, m}=\left(\frac{1}{n}+\frac{1}{m}\right)^{-1} \cdot\left[\frac{1}{n^{2}} \sum_{i=1}^{n} \sum_{j=1}^{m} \prod_{s=1}^{d}\left(1-U_{i s, n} \vee U_{j s, n}\right)+\right. \\
& +\frac{1}{m^{2}} \sum_{i=1}^{m} \sum_{j=1}^{m} \prod_{s=1}^{d}\left(1-V_{i s, m} \vee V_{j s, m}\right)- \\
& \left.-\frac{2}{n m} \sum_{i=1}^{n} \sum_{j=1}^{m} \prod_{s=1}^{d}\left(1-U_{i s, n} \vee V_{j s, m}\right)\right]
\end{aligned}
$$

where $u \vee v=\max (u, v)$.

\section{Bootstrap methods}

The bootstrap is a usually computer-intensive, resampling method for estimating the distribution of a statistic of interest. The concept of the bootstrap was introduced in the classical article by Bradley Efron (Efron, 1979) and, since then, it has become one of the most widely used Monte Carlo methods in a number of areas of applied sciences. 

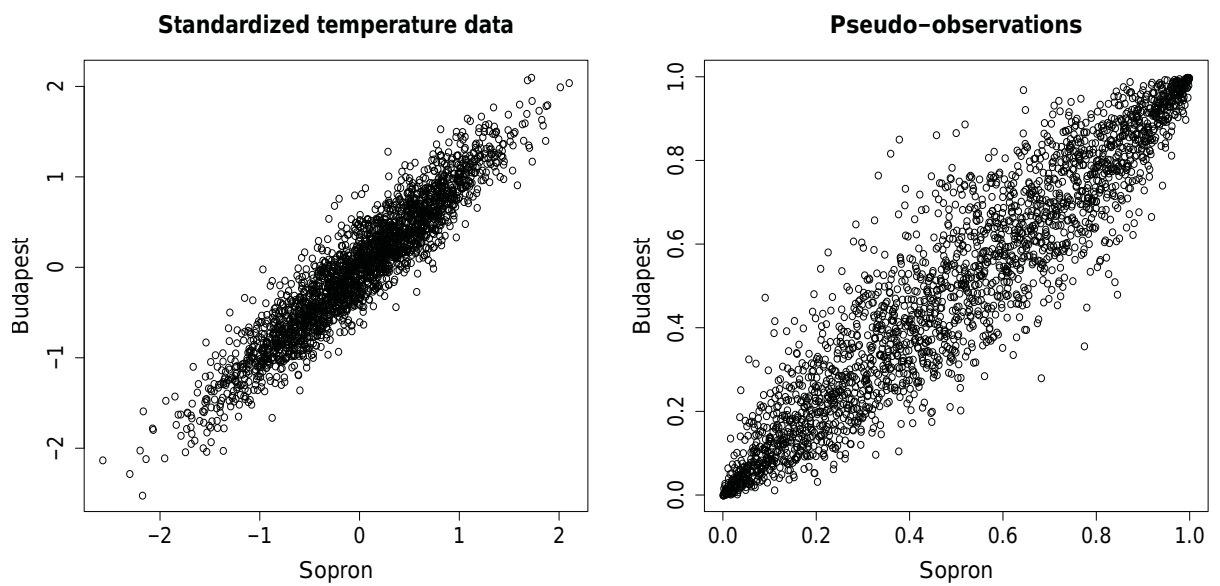

Figure 1. Bivariate data and the corresponding pseudo-observations.

\subsection{Bootstrap for i.i.d. data}

Let $\mathcal{X}_{n}=\left(X_{1}, \ldots, X_{n}\right)^{T}$ be a sequence of independent, identically distributed (i.i.d.) random variables with unknown common univariate distribution $F$ and let $T_{n}=t_{n}\left(\mathcal{X}_{n} ; F\right)$ be a statistic (like the sample mean $\bar{X}$ ). As $F$ is unknown, the distribution of the statistic $T_{n}$ is also unknown. Our main purpose is to approximate the distribution of $T_{n}$ or its function of interest - for example the standard deviation of $T_{n}$ (the standard error) or some of its quantiles for estimating $p$ values. The basic bootstrap method (mostly referred as i.i.d. bootstrap) is the following. For a given $\mathcal{X}_{n}$, we draw a random sample $\mathcal{X}_{m}^{*}=\left\{X_{1}^{*}, \ldots, X_{m}^{*}\right\}$ of size $m$ (usually $m=n$ ) with replacement from $\mathcal{X}_{n}$. Therefore, the common distribution of the $X_{i}^{*}$ is given by the empirical distribution $\hat{F}_{n}=$ $n^{-1} \sum_{i=1}^{n} \delta_{X_{i}}$, where $\delta_{z}$ is the probability measure having unit mass at $z$. In the next step, we define the bootstrap version of the statistic $T_{n}: T_{m, n}^{*}=t_{m}\left(\mathcal{X}_{m}^{*} ; \hat{F}_{n}\right)$. By repeating this procedure, we can approximate the unknown distribution $G_{n}$ of $T_{n}$ by its bootstrap counterpart $G_{n}^{*}$. In most of the cases the distribution of $G_{n}^{*}$ cannot be determined explicitly, but it can be approximated by simulation.

\subsection{Block bootstrap methods}

In our case we are interested in the effect of serial dependence on the homogeneity tests and on modelling in general, for example on the covariance matrix of our estimators. If the data are dependent then the estimates based on i.i.d. bootstrap methods may not be consistent.

In the presence of serial dependence, one of the most commonly used methods is the block bootstrap, see Lahiri (2003) for details. In this paper, we generalise the circular block bootstrap (CBB), which can be defined as follows. First, we wrap the data $X_{1}, \ldots, X_{n}$ around a circle, i.e. define the series $\widetilde{X}_{t}=X_{t_{\bmod (n)}}(t \in \mathbb{N})$, where $\bmod (n)$ denotes division "modulo $n$ ". This means that $X_{k}=\widetilde{X}_{k}=\widetilde{X}_{k+n}=\widetilde{X}_{k+2 n}=\ldots$ for

all $k \in\{1,2, \ldots, n\}$. For some $m$, let $i_{1}, \ldots, i_{m}$ be a uniform sample from the set $\{1,2, \ldots, n\}$. Then, for a given block size $b$, we construct $n^{\prime}=m \cdot b\left(n^{\prime} \approx n\right)$ pseudo-data:

$$
\begin{array}{r}
\widetilde{X}_{(k-1) b+j}^{*}=\widetilde{X}_{i_{k}+j-1} \text { where } j=1,2, \ldots, b \\
\text { and } k=1,2, \ldots, m .
\end{array}
$$

Finally, let us calculate the function of interest, for example the bootstrap sample mean as follows: $\widetilde{\widetilde{X}}_{n^{\prime}}^{*}=\frac{\widetilde{X}_{1}^{*}+\ldots+\widetilde{X}_{n^{\prime}}^{*}}{n^{\prime}}$. For the sake of simplicity we do not use this notation in the sequel; the asterisk simply denotes that the sample is a bootstrap sample.

Block length plays an important role in the process, and it is not trivial to determine its optimal value. Politis and White (2004) suggested an "automatic" block length selection algorithm (its correction was published in Patton et al., 2009) - but the practical applications of this method are far from obvious as there are parameters in it that have to be chosen.

We used a similar approach in our previous paper (Rakonczai et al., 2014). Our idea was that we tried to find the best block size by fitting a VAR model to the data and then checking the variance of $\bar{X}$ with the help of the block bootstrap. For sake of their simplicity, we restrict our attention to $\operatorname{VAR}(p)$ models. The methodology should be general enough to be compatible with other more complex classes of statistical models as well.

In Rakonczai et al. (2014), the block size was determined as the $\widehat{b}$, for which the estimated trace of the covariance matrix was closest to the one derived from the fitted VAR model:

$\widehat{b}=\underset{1 \leq b \in \mathbb{Z}}{\operatorname{argmin}}\left|\operatorname{tr}\left(\operatorname{Cov}\left(\overline{\boldsymbol{X}}_{\mathrm{VAR}}\right)\right)-\operatorname{tr}\left(\operatorname{Cov}_{*}\left(\overline{\boldsymbol{X}}_{b}^{*}\right)\right)\right|$,

where $\operatorname{Cov}_{*}\left(\overline{\boldsymbol{X}}_{b}^{*}\right)=\operatorname{Cov}\left(\overline{\boldsymbol{X}}_{b}^{*} \mid \mathcal{X}_{n}\right)$.

In the literature, simulations are naturally based on integer block sizes. But using the block length of Eq. (4), the estimated trace of covariance may be not be close enough to 
the theoretical trace of covariance. The same is true for other methods for block size determination. This may cause substantial bias, as in our case the relative difference between subsequent values of $\operatorname{tr}\left(\operatorname{Cov}_{*}\left(\overline{\boldsymbol{X}}_{b}^{*}\right)\right)$ can be quite large, especially for small $b$. This can be overcome by the following generalisation of the block bootstrap methodology.

\subsection{Generalised block bootstrap}

In case of $b>1, b \in \mathbb{R}$, let the generalised block bootstrap sample be defined as follows. Let $k$ be a random integer between 1 and the sample size $n$ and, again, let us wrap the sample around the circle. The bootstrap blocks are either of length $\lfloor b\rfloor$ or $\lceil b\rceil$ :

$$
\begin{array}{rrr}
X_{k}, X_{k+1}, \ldots, X_{k+\lfloor b\rfloor} \quad \text { with probability } & 1-b+\lfloor b\rfloor \\
X_{k}, X_{k+1}, \ldots, X_{k+\lceil b\rceil} & \text { with probability } & b-\lfloor b\rfloor,
\end{array}
$$

where $\lceil b\rceil$ denotes the upper and $\lfloor b\rfloor$ the lower integer part of $b$. At last, we put the blocks together. This procedure ensures that for integer-valued $b$ the new definition coincides with the traditional one, so this is indeed a generalisation. In the applications (Sect. 6) we show the clear advantages of this approach. Actually all relevant algorithms for finding the optimal block size can easily be adapted to find a solution in this generalised sense. In our case, instead of Eq. (4), we simply solve the equation in $b$

$$
\operatorname{tr}\left(\operatorname{Cov}\left(\overline{\boldsymbol{X}}_{\mathrm{VAR}}\right)\right)=\operatorname{tr}\left(\operatorname{Cov}_{*}\left(\overline{\boldsymbol{X}}_{b}^{*}\right)\right)
$$

In the same way as the circular block bootstrap sample, our generalised bootstrap sample is not a stationary process, conditional on the original sample. It is an important theoretical result of Politis and Romano (1994) that the stationary bootstrap sample is the only stationary block bootstrap sample - here the block lengths follow a geometric distribution, independent of each other.

The covariance matrix $\operatorname{Cov}_{*}\left(\overline{\boldsymbol{X}}_{b}^{*}\right)$ can be explicitly calculated. Henceforth, $P_{*}$ and $E_{*}$ denote the conditional distribution and the conditional expectation given the sample $\mathcal{X}_{n}$; therefore, $P_{*}\left(L_{1}=\lceil b\rceil\right)=P\left(L_{1}=\lceil b\rceil \mid \mathcal{X}_{n}\right)$ and $E_{*}\left(L_{1}\right)=$ $E\left(L_{1} \mid \mathcal{X}_{n}\right)$. Let us denote with $L_{1}, L_{2}, \ldots$ the block sizes - they are random variables independent of each other with common conditional distribution $P_{*}\left(L_{1}=\lceil b\rceil\right)=1-$ $P_{*}\left(L_{1}=\lfloor b\rfloor\right)=b-\lfloor b\rfloor$. We can also write $L_{i}=\lfloor b\rfloor+J_{i}$, where $J_{i} \mid \mathcal{X}_{n}$ follows a Bernoulli distribution with parameter $p=b-\lfloor b\rfloor$. Let $N$ be the random variable, which gives the number of blocks with block size $\lfloor b\rfloor$. If we have $N$, we can calculate the number of blocks with block size $\lceil b\rceil$, denoting it with $g(N)$. Therefore, $g(N)=\left\lfloor\frac{n-N \cdot\lfloor b\rfloor}{\lceil b\rceil}\right\rfloor$. Let us denote the remainder block size with $r(N)$, we can calculate it from the others: $r(N)=n-N \cdot\lfloor b\rfloor-g(N) \cdot\lceil b\rceil$. It can be seen that the conditional covariance matrix of the bootstrap mean can be calculated the following way:

$$
\begin{aligned}
& \operatorname{Cov}_{*}\left(\overline{\boldsymbol{X}}_{b}^{*}\right)=\frac{\lfloor b\rfloor^{2}}{n^{2}}\left[\operatorname{Cov}_{*}\left(\overline{\boldsymbol{X}}_{\lfloor b\rfloor, i}^{*}\right) \cdot E_{*} N+\operatorname{Cov}_{*} N \cdot \overline{\boldsymbol{X}}_{n}\left(\overline{\boldsymbol{X}}_{n}\right)^{T}\right]+ \\
& +\frac{\lceil b\rceil^{2}}{n^{2}}\left[\operatorname{Cov}_{*}\left(\overline{\boldsymbol{X}}_{\lceil b\rceil, i}^{*}\right) \cdot E_{*}(g(N))+\operatorname{Cov}_{*}(g(N)) \cdot \overline{\boldsymbol{X}}_{n}\left(\overline{\boldsymbol{X}}_{n}\right)^{T}\right]+ \\
& +\frac{1}{n^{2}}\left[\sum_{i=0}^{\lfloor b\rfloor} i^{2} P_{*}(r(N)=i) \cdot \operatorname{Cov}_{*}\left(\overline{\boldsymbol{X}}_{i, 1}^{*}\right)+\operatorname{Cov}_{*}(r(N)) \cdot \overline{\boldsymbol{X}}_{n}\left(\overline{\boldsymbol{X}}_{n}\right)^{T}\right] .
\end{aligned}
$$

We have to mention that the Politis and White algorithm actually gives a real number and not an integer as the optimal block size - this could be used without any rounding by our proposed method. In their original paper (Politis and White, 2004), the algorithm has been tested on data simulated from an AR(1) process and gave fair values for the optimal block length. However, when we tried to use this method to estimate the optimal block length for some meteorological data (wind speed and precipitation), the algorithm gave too large optimal block lengths.

Note as well that the type of block length that would be best for the block bootstrap method depends on the inference problem (e.g. variance estimation or testing), as described in Hall et al. (1995) or Lahiri (2003). There are two general strategies for block selection that can be applied to problems such as the homogeneity testing problem that we wish to consider. These block selection methods are described in Lahiri (2003) and Nordman and Lahiri (2014), based on either subsampling (Hall et al., 1995) or non-parametric plug-in (Lahiri et al., 2007). We think that these approaches can be modified for the proposed generalised block bootstrap, and we plan to investigate this possibility in a separate more theoretical paper.

\subsection{Algorithm for calculating $p$ value for homogeneity test of copulas}

As the limit distribution of the statistic $S_{n, m}$ is not distribution free, a simulation algorithm is needed to get critical values. The algorithm is the following:

1. Compute the statistic $S_{n, m}$, based on the original samples.

2. Generate $B$ generalised bootstrap samples $\mathcal{X}_{n}^{*(i)}=\left(\boldsymbol{X}_{1}^{*(i)}, \ldots, \boldsymbol{X}_{n}^{*(i)}\right)^{T} \quad i=1,2, \ldots, B$

from the first observation vector of size $n$.

3. Compute the statistics $S_{n, m}^{*(i)} \quad i=1,2, \ldots, B$, based on the bootstrap samples and the original second, $m$ length sample.

4. Compute the $p$ value: $\frac{1+\#\left\{S_{n, m}^{*(i)} \geq S_{n, m}\right\}}{B+1}$.

Guaranteeing the homogeneity of copulas in the context of bootstrap approaches is still an unsolved problem in general. Traditional bootstrap approaches have been claimed to 
be inconsistent for Cramér-von Mises statistics (Rémillard and Scaillet, 2009). The multiplier method of Rémillard and Scaillet (2009), which addresses the latter problem, is consistent but numerically costly, and thus practically not applicable to sample sizes of $O(1000)$ as considered in the present work. Martínez-Camblor et al. (2014) introduced a general bootstrap algorithm leading (under weak assumptions) to a consistent bootstrap statistic when testing for the homogeneity of the marginal distributions of a $k$-dimensional random variable; Huang and Jing (2014) suggested a special bootstrap method for a Cramér-von Mises test for the homogeneity of two distributions, which has a consistent limit distribution. However, these two recent developments are not directly transferable to the problem of testing for the homogeneity of two copulas. In the case of VAR(1) models as studied in the present work, numerical results indicate the homogeneity of the copulas under the employed generalised block bootstrap.

\section{Simulations}

In this section we present some properties of the copula homogeneity test obtained via simulations, strongly focusing on a specific $\operatorname{VAR}(p)$ process, arising in Sect. 6.

By using the bootstrap methodology, we can investigate the significance level of the homogeneity test. Our simulations indicate that the test is consistent for each block size and each relevant time series model. However, we find, that for $\operatorname{VAR}(p)$ processes, the distribution of the test statistic is different, if the first sample is generated via block bootstrap simulations. We will illustrate this via the VAR(1) process with parameters

$\mathbf{A}=\left(\begin{array}{cc}0.097 & 0.216 \\ -0.103 & 0.403\end{array}\right)$ and $\mathbf{C}=\left(\begin{array}{cc}0.449 & 0.406 \\ 0.406 & 0.436\end{array}\right)$

let us call the VAR(1) process simulated with these parameters the "Budapest and Apatovac process", as these $A$ and $C$ matrices are the $\operatorname{VAR}(p)$ coefficient estimates of temperature data pairs for Budapest and Apatovac, as described in Sect. 6 . Table 1 shows that the empirical mean and the 0.9,0.95 and 0.99 empirical quantiles of the test statistic are substantially greater, if the first sample is bootstrapped, but essentially unaffected by the block size. So we found that the distribution of the bootstrapped test statistic is not the same as the one without bootstrapping. This is an interesting result, worthy of further investigation. Therefore, the reference distribution for our hypothesis testing was the empirical distribution of the generalised block bootstrap procedure, which depends on the underlying stochastic process and also the sample size.

Now we estimate the power of the proposed homogeneity test. We take samples from the VAR(1) process of $\mathrm{Bu}$ dapest and Apatovac - these are the fixed, $H_{0}$ samples. As alternative, $H_{1}$ hypothesis, we chose several models: $\operatorname{VAR}(p)$ models, but with other parameters, representing stronger dependence; i.i.d. bivariate normal distributed samples; MA(4)
Table 1. Simulated quantiles of the homogeneity test statistic for the VAR(1) process approximating the temperature data close to Budapest and Apatovac with sample size $n=1000$. The first row presents the simulation results without using the bootstrap.

\begin{tabular}{r|r|rrr}
\hline $\begin{array}{r}\text { Block } \\
\text { size }\end{array}$ & Mean & \multicolumn{3}{|c}{ Quantiles } \\
\hline- & 0.00629 & 0.0085 & 0.0093 & 0.0110 \\
\hline 1 & 0.00829 & 0.0124 & 0.0142 & 0.0178 \\
1.5 & 0.00823 & 0.0121 & 0.0141 & 0.0184 \\
2 & 0.00825 & 0.0124 & 0.0140 & 0.0181 \\
2.5 & 0.00824 & 0.0122 & 0.0140 & 0.0177 \\
3 & 0.00834 & 0.0125 & 0.0144 & 0.0190 \\
5 & 0.00821 & 0.0122 & 0.0139 & 0.0174 \\
5.5 & 0.00828 & 0.0122 & 0.0139 & 0.0179 \\
6 & 0.00832 & 0.0125 & 0.0144 & 0.0186 \\
7 & 0.00829 & 0.0124 & 0.0142 & 0.0178 \\
8 & 0.00831 & 0.0125 & 0.0143 & 0.0181 \\
8.5 & 0.00822 & 0.0121 & 0.0139 & 0.0178 \\
9 & 0.00839 & 0.0124 & 0.0142 & 0.0185 \\
10 & 0.00823 & 0.0123 & 0.0140 & 0.0184 \\
11 & 0.00824 & 0.0123 & 0.0140 & 0.0182 \\
12 & 0.00826 & 0.0123 & 0.0143 & 0.0180 \\
15 & 0.00831 & 0.0125 & 0.0142 & 0.0187 \\
\hline
\end{tabular}

models; mixtures of MA(4) models. In each case, the power of the test seems to converge to $100 \%$, as the sample size increases. For example, Table 2 shows powers for different block sizes, if the alternative hypothesis is a VAR(1) process with parameters

$\widetilde{\mathbf{A}}=\left(\begin{array}{ll}-0.1 & 0.3 \\ -0.8 & 0.9\end{array}\right)$ and $\widetilde{\mathbf{C}}=\left(\begin{array}{ll}0.449 & 0.406 \\ 0.406 & 0.436\end{array}\right)$.

The block size does not have a big effect on the power. We have to mention that although the numbers manifest consistency, the last digit of the powers in the table may not be accurate. We took 100 samples with 100 bootstrap replicates (let us call these $100 \times 100$ simulations) and as we made another $100 \times 100$ simulations, the powers sometimes differed by several percentages. This was especially true for the $99 \%$ significance level, therefore in the following, we do not show the simulations for this level.

As we shall see in the next section, for the data pairs Budapest and Apatovac, the block size of 8.71 is going to be a good choice. Table 3 shows that the tests are consistent also for this block length, using $100 \times 100$ simulations. It is important to note that these results are power values for a specific null and alternative hypothesis (typical for non-parametric statistical tests).

We also simulated the test statistic for other models: if the reference $H_{0}$ sample is

- i.i.d. bivariate normal distributed;

- an MA(4) process; 
Table 2. The power of the homogeneity test (\%). Null hypothesis: the VAR(1) process of Budapest and Apatovac for different block sizes and sample sizes $(n)$. Alternative hypothesis: also a VAR(1) process, but with other parameters, representing stronger dependence.

\begin{tabular}{r|ccc|ccc|ccc|cc|cc}
\hline & \multicolumn{3}{|c|}{$n=100$} & \multicolumn{3}{c|}{$n=500$} & \multicolumn{3}{c|}{$n=800$} & \multicolumn{3}{c}{$n=1186$} \\
\hline $\begin{array}{r}\text { Block } \\
\text { size }\end{array}$ & \multicolumn{3}{|c|}{ Confidence level } & \multicolumn{3}{|c|}{ Confidence level } & \multicolumn{2}{c|}{ Confidence level } & \multicolumn{3}{c}{ Confidence level } \\
& 90 & $95 \%$ & $99 \%$ & $90 \%$ & $95 \%$ & $99 \%$ & $90 \%$ & $95 \%$ & $99 \%$ & $90 \%$ & $95 \%$ & $99 \%$ \\
\hline 1 & 37.13 & 25.27 & 11.72 & 77.12 & 69.20 & 48.12 & 92.54 & 88.25 & 72.51 & 98.96 & 97.84 & 91.72 \\
1.5 & 36.50 & 24.86 & 11.87 & 77.44 & 69.12 & 45.84 & 92.27 & 87.58 & 74.16 & 98.65 & 97.16 & 90.48 \\
2 & 35.61 & 25.43 & 11.61 & 77.00 & 66.76 & 47.2 & 92.65 & 87.43 & 71.69 & 98.52 & 97.08 & 90.20 \\
5 & 32.54 & 22.48 & 8.95 & 77.48 & 67.68 & 43.16 & 92.61 & 86.62 & 72.40 & 98.56 & 96.68 & 89.96 \\
6 & 32.63 & 22.39 & 9.08 & 77.96 & 68.60 & 48.12 & 92.06 & 87.72 & 70.62 & 98.68 & 97.68 & 90.92 \\
6.5 & 33.47 & 22.90 & 9.15 & 77.64 & 68.88 & 50.56 & 91.83 & 87.69 & 74.17 & 98.38 & 97.16 & 91.43 \\
7 & 33.72 & 23.16 & 9.90 & 76.84 & 66.56 & 48.44 & 92.78 & 86.43 & 71.62 & 98.12 & 96.88 & 91.40 \\
7.5 & 32.68 & 22.14 & 8.91 & 77.84 & 69.20 & 46.48 & 92.18 & 86.90 & 73.89 & 98.39 & 97.16 & 90.02 \\
8 & 32.93 & 22.39 & 8.63 & 78.12 & 68.92 & 48.92 & 93.28 & 87.11 & 75.81 & 98.48 & 97.32 & 87.24 \\
8.5 & 33.49 & 23.01 & 9.39 & 78.56 & 69.52 & 48.84 & 93.37 & 87.02 & 74.47 & 98.49 & 97.16 & 91.85 \\
9 & 33.00 & 22.84 & 10.13 & 76.24 & 66.88 & 43.72 & 91.99 & 88.24 & 73.48 & 98.48 & 96.88 & 90.08 \\
9.5 & 33.41 & 21.83 & 8.30 & 76.92 & 66.60 & 45.00 & 92.24 & 87.91 & 72.06 & 98.31 & 97.16 & 91.64 \\
10 & 33.40 & 23.19 & 9.46 & 78.28 & 69.28 & 44.04 & 92.44 & 87.00 & 69.80 & 98.44 & 96.96 & 90.52 \\
11 & 32.69 & 22.65 & 8.38 & 75.92 & 66.96 & 48.36 & 92.48 & 87.72 & 74.16 & 98.16 & 96.88 & 92.12 \\
12 & 32.80 & 22.04 & 9.66 & 77.04 & 68.00 & 44.16 & 92.04 & 88.20 & 75.28 & 98.28 & 97.16 & 90.00 \\
13 & 31.68 & 21.84 & 8.53 & 77.04 & 69.28 & 48.96 & 92.80 & 88.28 & 76.28 & 98.12 & 96.68 & 88.72 \\
14 & 31.98 & 22.24 & 9.32 & 77.84 & 68.40 & 47.56 & 92.52 & 88.48 & 72.56 & 98.60 & 96.24 & 89.12 \\
15 & 32.33 & 22.65 & 8.44 & 77.20 & 66.84 & 42.80 & 91.64 & 86.84 & 71.00 & 98.56 & 96.68 & 88.24 \\
\hline
\end{tabular}

Table 3. The power of the homogeneity test $(\%)$. Null hypothesis: the $\operatorname{VAR}(1)$ process of Budapest and Apatovac $\left(H_{0}\right)$ for block size 8.71; against the VAR(1) process simulated with coefficient matrices $\widetilde{\mathbf{A}}$ and $\widetilde{\mathbf{C}}$ as alternative hypothesis.

\begin{tabular}{r|cc|c|cc}
\hline Sample & \multicolumn{2}{|c|}{ Confidence level } & Sample & \multicolumn{2}{c}{ Confidence level } \\
size & $90 \%$ & $95 \%$ & size & $90 \%$ & $95 \%$ \\
\hline 50 & 22.44 & 14.76 & 500 & 81.28 & 74.28 \\
100 & 34.04 & 24.76 & 800 & 92.80 & 89.04 \\
200 & 48.04 & 37.04 & 1000 & 96.80 & 93.84 \\
300 & 64.92 & 55.04 & 1200 & 98.96 & 98.08 \\
400 & 74.68 & 65.08 & 1500 & 99.92 & 99.88 \\
\hline
\end{tabular}

- a stationary GARCH(1,1) process.

In the two latter cases, we simulated samples for two cases in which the coordinates were independent and correlated, respectively. The alternative hypothesis varied from test to test, for example if the $H_{0}$ sample was i.i.d. bivariate normal distributed, we chose $H_{1}$ as another i.i.d. bivariate normal distributed process and a VAR(1) process. Each homogeneity test proved to be consistent. Table 4 shows the power of the homogeneity test, when the coordinates of the reference sample are a realisation of a $\operatorname{GARCH}(1,1)$ process with parameters $0.001,0.028$ and 0.97 - typical values if we model exchange rates with the $\operatorname{GARCH}(1,1)$ process. The alternative hypothesis was a VAR(1) process. In this case the fractional block size had a bit of a stronger effect on the powers of the test and the block size 11.35 proved to be the most
Table 4. The power of the homogeneity test (\%). Null hypothesis: $\operatorname{GARCH}(1,1)$ process with different block sizes, with sample size $n=100$. Alternative hypothesis: VAR(1) process simulated with coefficient matrices $\mathbf{A}$ and $\mathbf{C}$.

\begin{tabular}{r|cc|c|cc}
\hline Block & \multicolumn{2}{|c|}{ Confidence level } & Block & \multicolumn{2}{c}{ Confidence level } \\
size & $90 \%$ & $95 \%$ & size & $90 \%$ & $95 \%$ \\
\hline 1 & 60.13 & 47.41 & 8 & 61.04 & 45.15 \\
1.25 & 60.12 & 46.41 & 10 & 60.82 & 46.69 \\
1.5 & 60.53 & 46.53 & 11 & 63.08 & 47.26 \\
1.75 & 61.07 & 45.63 & 11.25 & 63.13 & 47.94 \\
2 & 61.28 & 48.01 & 11.35 & 63.67 & 48.29 \\
3 & 59.92 & 47.00 & 11.75 & 63.36 & 47.57 \\
4 & 62.20 & 46.39 & 12 & 62.19 & 46.32 \\
5 & 61.14 & 46.63 & 12 & 60.86 & 46.83 \\
6 & 61.30 & 46.69 & 13 & 60.77 & 45.95 \\
7 & 59.91 & 44.57 & 15 & 61.06 & 46.02 \\
\hline
\end{tabular}

powerful. This was the only example in which we found a specific block size, which essentially maximised the power of our homogeneity test.

\section{Applications}

The observations comprise 63 years of daily temperature data of the European Climate Assessment (E-OBS; http://www. ecad.eu). The methodology of deriving the data for the grid points has been published in Haylock et al. (2008), where this database has been used extensively for climate analysis. 


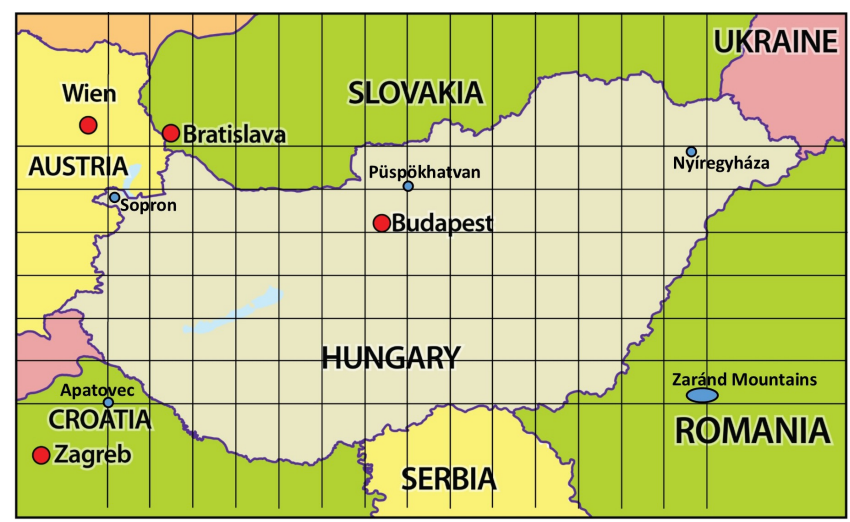

Figure 2. The map of the Carpathian Basin with the used grid points.

We have worked with the part of the $0.5^{\circ}$ grid - available for whole Europe and northern Africa - which lies in the Carpathian Basin. Figure 2 depicts the used grid points. For later reference, we chose the grid point next to Budapest, one grid point in the neighbourhood of the Hungarian capital and four further grid points lying far from Budapest, in different directions.

The quality of the data has been evaluated, e.g., in Hofstra et al. (2009), and it turned out to be reliable for most of central Europe. As we have used the grid points, belonging to the Carpathian Basin, this validates our data.

As we intend to use models, suitable for stationary data, first the stationarity had to be ensured. We have first subtracted the smoothed daily averages from the observations. The smoothing was made by loess regression; Fig. $3 \mathrm{a}$, b depict the daily averages and standard deviations of the 63-year data and the smoothing regression line for the grid point near Budapest. It turned out that the second-order stationarity assumption is still far from being true (in winter the variances were substantially larger than in summer), so we have divided the observations by the smoothed estimated standard deviation for the given day:

$\tilde{x}_{t, n}=\frac{x_{t, n}-m_{t}}{s_{t}}$,

where $\widetilde{x}_{t, n}$ is the standardised value for day $t$ in year $n$, based on the original observation $x_{t, n}$ for the same day and the smoothed average $m_{t}$ and smoothed standard deviation $s_{t}$. Figure $3 \mathrm{c}$ shows the original daily observations and the standardised data between 1 January 2010 and 31 December 2012 for the grid point near Budapest.

In order to reduce the strong serial dependence, we have finally computed the 10 -day averages of the $\tilde{x}$ values. As there are no outliers in the temperature data (see Fig. 3c) and the series is nearly normal, the mean was chosen as the most suitable function. There is a slight but significant upward linear trend in the data, but we did not remove it, as one of our main aims was to detect the changes in the dependencies of the in- vestigated sites - and these should be based on the original (standardised) deviations, as constructed above.

In the next step, we examined the fixed grid point near Budapest paired with other grid points of the database. Using the Akaike information criterion, we chose the orders of the most appropriate vector autoregression to model our data pairs. Despite the adjusted $R^{2}$ values being rather low (around $10 \%$ ), the Ljung-Box Q test, the Breusch-Godfrey test and the test of Kojadinovic and Yan (2011a) could not detect the presence of further serial dependence that has not been included in the VAR model. Table 5 contains these results.

Our main goal is to detect if there is a significant change in the dependence structure of the data. We separated the pairs of points into two parts - the first part corresponds to the first 31.5 years' observations and the second part to the second 31.5 years' observations. For five selected pairs of grid points, we wanted to test the null hypothesis that the copula of first half of the sample is equal to the copula of the second half of the sample. We have tested the independence by the Cramér-von Mises type test of Kojadinovic and Holmes (2009), included in the R package copula and the result was clear - the test could not detect any dependence (neither for the used complete halves, nor for shorter adjacent subsequences from sample sizes of 100 to 1000).

Table 6 and Fig. 4 depict the optimal block lengths obtained from solving Eq. (5). The second column of Table 6 and the red line of Fig. 4 show the trace of the covariance matrices of the mean, calculated from the fitted VAR(1) models, multiplied by 1186 - the half of the original sample size. In Fig. 4a it can be seen that the traces of the covariance matrix of the mean at the integer block sizes 13 and 14 are 17.8 and 18.05 (green dashed lines), which are quite far from the red line. Figure $4 \mathrm{~b}$ shows that the trace is not monotonic in the neighbourhood of the optimum, so we have to be cautious when we search for the optimal block size, because Eq. (5) can have multiple solutions. The trace function (black line in Fig. 4) is always continuous, but not necessarily differentiable, resulting from the construction of our generalised block bootstrap method. This follows from Eq. (6), where there are lower and upper integer parts of $b$ in the formula.

Generally, we noticed, that as the block sizes tend to be smaller, the trace function is closer to be monotonic. This phenomenon can be explained by the expansion of $\operatorname{Cov}_{*}\left(\overline{\boldsymbol{X}}_{b}^{*}\right)$ in formula (6): if the block size is relatively small compared to the sample size, then the first and second terms are much more dominant over the third part - which contains the effect of the remainder block size. We got pretty small, 8.71 optimal block size for the pairs Budapest and Apatovac, and a much larger value of 55.42 for Budapest and Zaránd Mountains. This reflects that the optimal lag order of the fitted VAR(1) model was much higher for the pairs Budapest and Zaránd Mountains. In case of the five selected pairs, as many as seven iterations were always enough to solve Eq. (5). We have to mention that there exist some pairs of grid points - 
(a) Daily averages of the data, Budapest

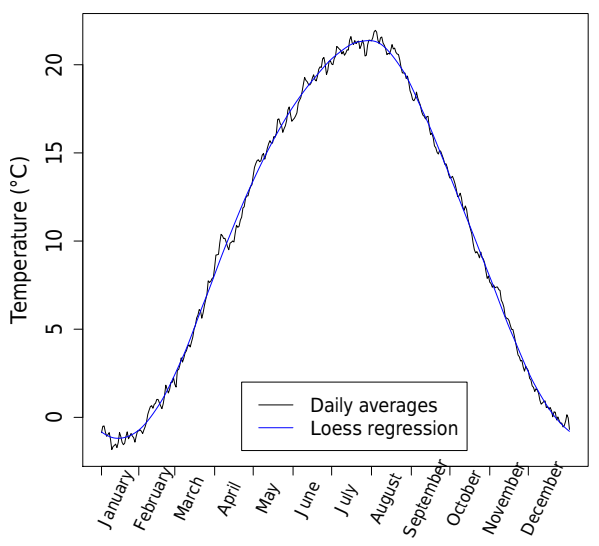

(b) Daily standard deviations, Budapest

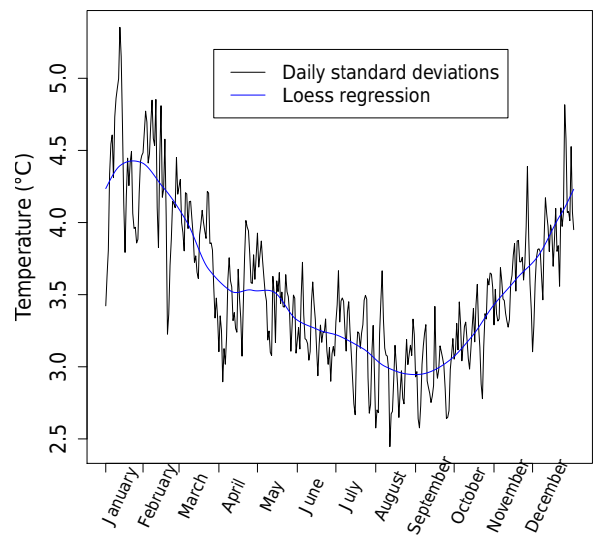

(c) Daily observations, Budapest

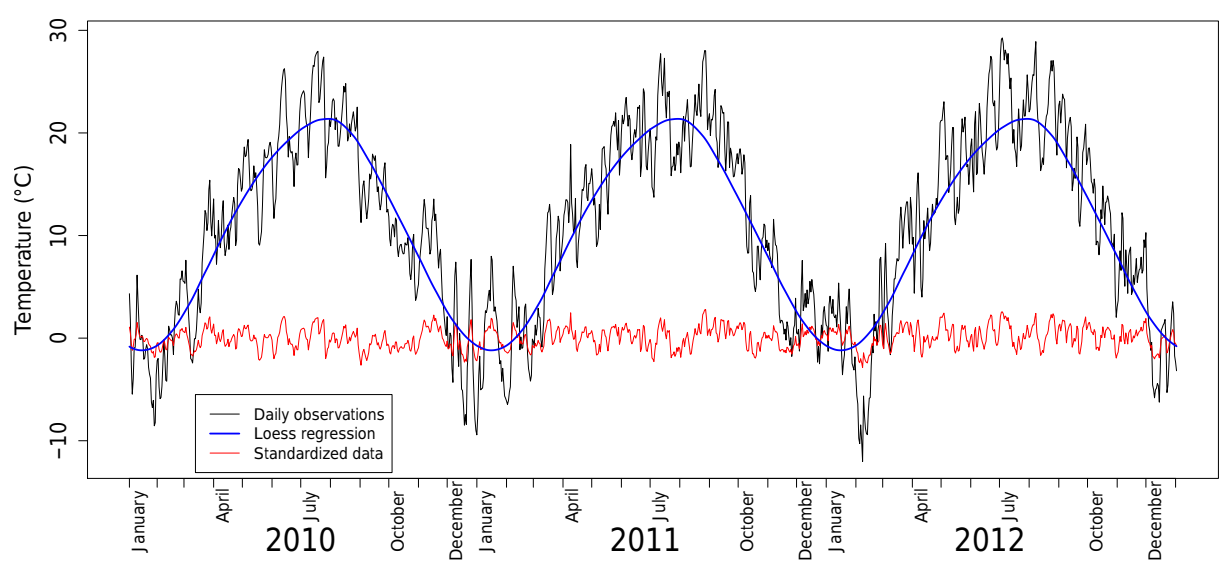

Figure 3. (a) The daily averages (annual cycle) and the smoothing loess regression; (b) the daily standard deviations (annual cycle) and the smoothing loess regression; (c) the original and the standardised data for the grid point near Budapest.

Table 5. Results of tests checking for serial dependence between the estimated residuals ( $p$ values) and the chosen order of the VAR model by Akaike criterion.

\begin{tabular}{l|c|c|c|c}
\hline Pairs of selected grid points & $\begin{array}{c}\text { Optimal } \\
\text { order }\end{array}$ & $\begin{array}{c}\text { Ljung- } \\
\text { Box }\end{array}$ & $\begin{array}{c}\text { Breusch- } \\
\text { Godfrey }\end{array}$ & $\begin{array}{c}\text { Genest-Rémillard- } \\
\text { Kojadinovic-Yan }\end{array}$ \\
\hline Budapest and Sopron & 3 & 0.271 & 0.324 & 0.259 \\
Budapest and Apatovac & 1 & 0.080 & 0.463 & 0.120 \\
Budapest and Zaránd Mountains & 9 & 0.174 & 0.017 & 0.149 \\
Budapest and Nyíregyháza & 4 & 0.174 & 0.474 & 0.258 \\
Budapest and Püspökhatvan & 4 & 0.155 & 0.539 & 0.276 \\
\hline
\end{tabular}

especially at the southern part of the Carpathian Basin - for which Eq. (5) is not solvable.

The last step was conducting the copula homogeneity test described in Sect. 3. Using the optimal block size, we generated bootstrap samples via the generalised block bootstrap method for the first half of the sample. With the empirical copulas of these bootstrap samples and the empirical copula of the second part of the original sample, we can calculate the test statistic $S_{n, m}$. In our case $n=m=1186$. In order to get accurate $p$ values, we used $10^{4}$ repetitions. This procedure can be conducted in the reversed way as well: we may generate bootstrap samples from the second half of the sample and fix the first half. Table 7 contains the results of the homogeneity test for both versions. The dependence structure proved to be different in the first 31.5 years at the two pairs Budapest and Apatovac and Budapest and Zaránd Mountains. Figure 5 depicts the 10-day averages of the standardised observations and their copula of the pair Budapest and Apatovac. We can 
(a) Budapest \& Sopron

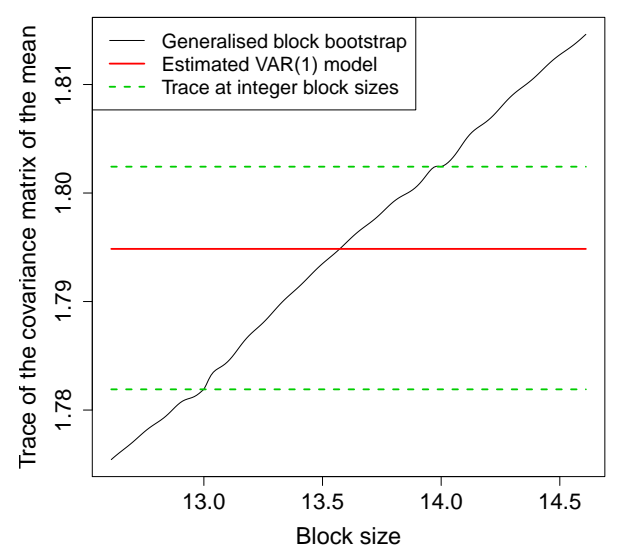

(b) Budapest \& Apatovac

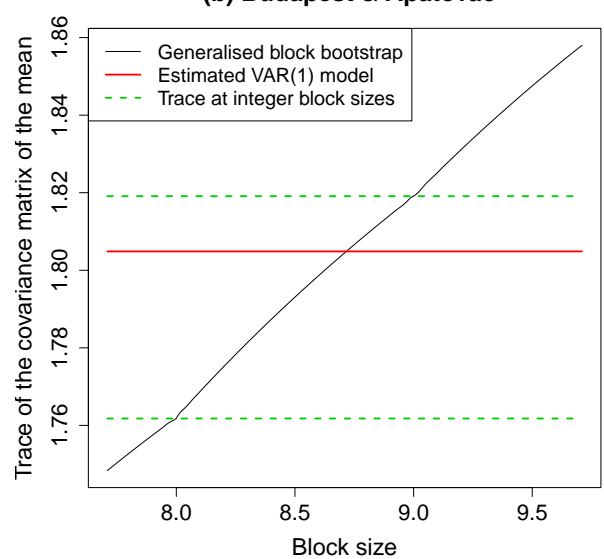

Figure 4. (a) The trace of the covariance matrix of the mean for two selected grid points near Budapest and Sopron; (b) the trace of the covariance matrix of the mean for two selected grid points near Budapest and Apatovac (first half of the sample).

Table 6. Optimal block length for the first half of the samples for the five selected pairs of grid points.

\begin{tabular}{l|c|c|c}
\hline Pairs of selected grid points & $n \cdot \operatorname{tr}\left(\operatorname{Cov}\left(\overline{\boldsymbol{X}}_{\text {VAR }}\right)\right)$ & $\begin{array}{c}\text { Optimal } \\
\text { block size }\end{array}$ & $\begin{array}{c}\text { Number of } \\
\text { iterations }\end{array}$ \\
\hline Budapest and Sopron & 1.759 & 13.61 & 2 \\
Budapest and Apatovac & 1.805 & 8.71 & 2 \\
Budapest and Zaránd Mountains & 4.427 & 55.42 & 7 \\
Budapest and Nyíregyháza & 1.879 & 9.88 & 3 \\
Budapest and Püspökhatvan & 2.140 & 15.68 & 3 \\
\hline
\end{tabular}

Table 7. $p$ Values of the copula homogeneity test, if the first/second half of the sample is bootstrapped - based on $10^{4}$ simulations.

\begin{tabular}{l|c|c}
\hline \multirow{2}{*}{ Pairs of selected grid points } & \multicolumn{2}{|c}{$p$ values, bootstrapped half: } \\
& first & second \\
\hline Budapest and Sopron & 0.064 & 0.047 \\
Budapest and Apatovac & 0.028 & 0.012 \\
Budapest and Zaránd Mountains & 0.034 & 0.042 \\
Budapest and Nyíregyháza & 0.116 & 0.062 \\
Budapest and Püspökhatvan & 0.848 & 0.751 \\
\hline
\end{tabular}

see that the pseudo-observations are apparently somewhat different, and the test also detected deviance between the two copulas.

\section{Conclusions}

In this paper, we have used the bootstrap for determining the $p$ values of a homogeneity test for copulas. But the approach of block bootstrap is much more generally applicable than in our case. Our results underline that the block size determination is definitely not yet a completely solved question in spite of the available asymptotic results, as for finite samples and different statistical inference or testing problems the results might also be substantially different.

We can summarise our findings as follows.

First, we proposed a simple generalisation of the block bootstrap methodology, which fits naturally to the existing algorithms, and which helps to overcome the problem of discreteness in the usual block size. The proposed generalised block bootstrap method can easily be applied to any other problem, where the block size plays an important role, as all block length determining algorithms give a real number as estimated block size.

Second, we have found some significant changes in the dependence structure between the standardised temperature values of pairs of stations within the Carpathian Basin. The direction of this change may be worth further investigation, as this may lead to a better understanding of the recent changes in our climate.

It is an interesting open question, to which models and inference problems the proposed block size determining method - based on functions of the variances - can be successfully applied. We have checked by simulation that the method can be applied to the specific VAR models, described in our article. For non-linear time series we might need more observations to get a fit, which is similarly reliable. As a general comment on the use of bootstrap methods, we have seen 
Pseudo-observations, first half of the sample

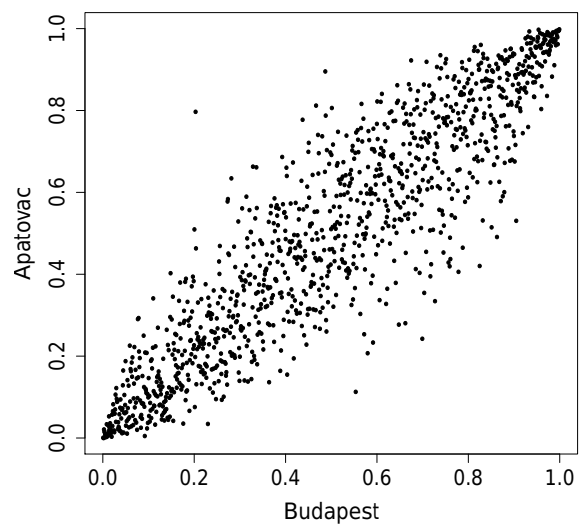

Pseudo-observations, second half of the sample

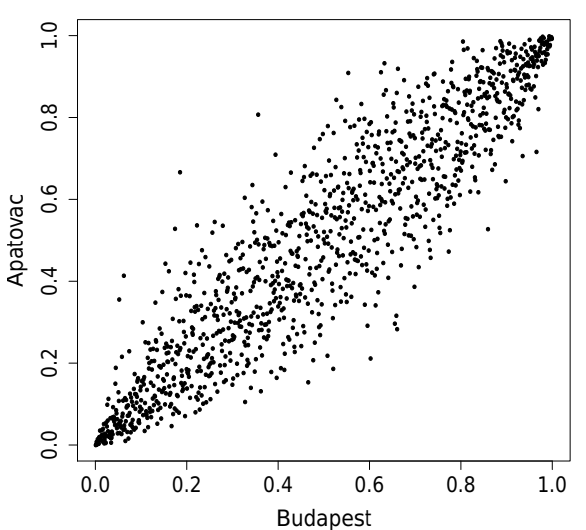

Figure 5. The pseudo-observations of Budapest and Apatovac for the first and the second half of the 10-day averages of the standardised observations.

cases when the block size did not play an important role, but in our opinion this is rather an exception than a rule. Choosing a not optimal block size may decrease the accuracy of the applied method somewhat, but not using any type of block bootstrap may distort the results completely as quite a few of the references have already demonstrated.

Data availability. The E-OBS dataset is regularly refreshed. The most up-to-date version is 15.0, dated June 2017. However at the time of the writing of the paper, 12.0 was the most recent, which is still available from the webpage http://www.ecad.eu/download/ ensembles/oldversions.php.

Competing interests. The authors declare that they have no conflict of interest.

Acknowledgements. We acknowledge the E-OBS data set from the EU-FP6 project ENSEMBLES (http: //ensembles-eu.metoffice.com) and the data providers in the ECA\&D project (http://www.ecad.eu).

Edited by: R. Donner

Reviewed by: three anonymous referees

\section{References}

Brockwell, P. J. and Davis, R. A.: Time series: theory and methods, Springer Science \& Business Media, 2013.

Bücher, A. and Volgushev, S.: Empirical and sequential empirical copula processes under serial dependence, J. Multivariate Anal., 119, 61-70, 2013.

Chernick, M. R.: Bootstrap methods: A guide for practitioners and researchers, vol. 619, John Wiley \& Sons, 2011.
Cong, R.-G. and Brady, M.: The interdependence between rainfall and temperature: copula analyses, The Scientific World Journal, 405675, https://doi.org/10.1100/2012/405675, 2012.

Efron, B.: Bootstrap methods: another look at the jackknife, Ann. Stat., 7, 1-26, 1979.

Farook, A. J. and Kannan, K. S.: Climate Change Impact on Rice Yield in India-Vector Autoregression Approach, Sri Lankan Journal of Applied Statistics, 16, 161-178, 2016.

Genest, C. and Rémillard, B.: Test of independence and randomness based on the empirical copula process, Test, 13, 335-369, 2004.

Genest, C., Quessy, J.-F., and Rémillard, B.: Goodness-of-fit procedures for copula models based on the probability integral transformation, Scand. J. Stat., 33, 337-366, 2006.

Hall, P., Horowitz, J. L., and Jing, B.-Y.: On blocking rules for the bootstrap with dependent data, Biometrika, 82, 561-574, 1995.

Haylock, M., Hofstra, N., Klein Tank, A. M. G., Klok, E. J., Jones, P. D., and New, M.: A European daily highresolution gridded data set of surface temperature and precipitation for 1950-2006, J. Geophys. Res.-Atmos., 113, D20, https://doi.org/10.1029/2008JD010201, 2008.

Hill, D., Bell, K. R. W., McMillan, D., and Infield, D.: A vector auto-regressive model for onshore and offshore wind synthesis incorporating meteorological model information, Adv. Sci. Res., 11, 35-39, https://doi.org/10.5194/asr-11-35-2014, 2014.

Hoeffding, W.: Massstabinvariante korrelationstheorie, Teubner, 1940.

Hofstra, N., Haylock, M., New, M., and Jones, P. D.: Testing EOBS European high-resolution gridded data set of daily precipitation and surface temperature, J. Geophys. Res.-Atmos., 114, D21, https://doi.org/10.1029/2009JD011799, 2009.

Huang, Q. and Jing, P.: Cramer-Von Mises Statistics for Testing the Equality of Two Distributions, in: Frontier and Future Development of Information Technology in Medicine and Education, Springer, 93-101, 2014.

Kojadinovic, I. and Holmes, M.: Tests of independence among continuous random vectors based on Cramér-von Mises functionals of the empirical copula process, J. Multivariate Anal., 100, 11371154, 2009. 
Kojadinovic, I. and Yan, J.: Tests of serial independence for continuous multivariate time series based on a Möbius decomposition of the independence empirical copula process, Ann. I. Stati. Math., 63, 347-373, $2011 \mathrm{a}$.

Kojadinovic, I. and Yan, J.: A goodness-of-fit test for multivariate multiparameter copulas based on multiplier central limit theorems, Stat. Comput., 21, 17-30, 2011 b.

Lahiri, S. N.: Resampling methods for dependent data, Springer Science \& Business Media, 2003.

Lahiri, S. N., Furukawa, K., and Lee, Y.-D.: A nonparametric plugin rule for selecting optimal block lengths for block bootstrap methods, Statistical Methodology, 4, 292-321, 2007.

Martínez-Camblor, P., Carleos, C., and Corral, N.: Cramér-von mises statistic for repeated measures, Revista Colombiana de Estadística, 37, 45-67, 2014.

Nelsen, R. B.: An introduction to copulas, Springer Science \& Business Media, 2007.

Nordman, D. J. and Lahiri, S. N.: Convergence rates of empirical block length selectors for block bootstrap, Bernoulli, 20, 958978, 2014

Norrulashikin, S. M., Yusof, F., and Kane, I. L.: An Investigation towards the Suitability of Vector Autoregressive Approach on Modeling Meteorological Data, Modern Applied Science, 9, 89$100,2015$.
Patton, A., Politis, D. N., and White, H.: Correction to "Automatic block-length selection for the dependent bootstrap" by D. Politis and H. White, Economet. Rev., 28, 372-375, 2009.

Politis, D. N. and Romano, J. P.: The stationary bootstrap, J. Am. Stat. Assoc., 89, 1303-1313, 1994.

Politis, D. N. and White, H.: Automatic block-length selection for the dependent bootstrap, Economet. Rev., 23, 53-70, 2004.

Rakonczai, P., Varga, L., and Zempléni, A.: Copula fitting to autocorrelated data with applications to wind speed modelling, Annales Universitatis Scientarium de Rolando Eotvos Nominatae, Sectio Computatorica, 43, 3-20, 2014.

Rémillard, B. and Scaillet, O.: Testing for equality between two copulas, J. Multivariate Anal., 100, 377-386, 2009.

Schölzel, C. and Friederichs, P.: Multivariate non-normally distributed random variables in climate research - introduction to the copula approach, Nonlin. Processes Geophys., 15, 761-772, https://doi.org/10.5194/npg-15-761-2008, 2008.

Shumway, R. H. and Stoffer, D. S.: Time series analysis and its applications with R examples, Springer Science \& Business Media, 2011.

Sims, C. A.: Macroeconomics and reality, Econometrica: Journal of the Econometric Society, 1-48, 1980. 